\title{
Large Malignant Peripheral Nerve Sheath Tumor of Lumbar spine: A Case Report
}

\author{
Lamichhane B, ${ }^{1}$ Yogi N, ${ }^{1}$ Paudel GR, ${ }^{1^{*}}$ Panth $R,{ }^{2}$ Devkota UP, ${ }^{1}$ Gongal DN ${ }^{1}$ \\ ${ }^{1}$ Department of Neurosurgery, ${ }^{2}$ Department of Pathology, National Institute of \\ Neurological and Allied Sciences, Bansbari, Kathmandu, Nepal
}

\author{
*Corresponding Author: \\ Gun Raj Paudel, MBBS, MS \\ MCh Resident \\ Department of Neurosurgery \\ National Institute of Neurological and Allied Sciences \\ Email: drgunrajpaudel@gmail.com

\section{Citation} \\ Lamichhane B, Yogi N, Paudel GR, Panth R, Devkota UP, \\ Gongal DN. Large Malignant Peripheral Nerve Sheath \\ Tumor of Lumbar spine: A Case Report. Nepal Journal of \\ Medical Sciences 2013;2(2):190-3.
}

\begin{abstract}
Malignant peripheral nerve sheath tumors (MPNST) is a rare condition that arises from the peripheral nerves and is characterized histologically by cellular atypia, hyperchromasia and spindle shaped tumor cells intersecting in stroiform pattern. While neurofibromas are common in spinal canal, malignant transformation into MPNST is rarely seen except in cases with neurofibromatosis 1 (NF1).
\end{abstract}

Keywords: Malignant Peripheral nerve sheath tumor; neurofibroma; neurofibromatosis 1

\section{Introduction:}

Malignant peripheral nerve sheath tumors (MPNSTs) represent a rare variety of soft tissue sarcomas involving major or minor peripheral nerve branches, which are thought to originate from Schwann cells or from pluripotent cells of the neural crest. It was formerly referred to as malignant schwannomas, malignant neurilemmomas, neurogenic sarcomas, or neurofibrosarcomas, and can account for up to $10 \%-12 \%$ of soft tissue sarcomas. It is a rare tumor. The incidence of MPNST arising in neurofibromatosis is $4.6 \%$ and $0.001 \%$ in the general population. ${ }^{1}$ Neurofibromas are although common benign masses of the spinal canal, malignant degeneration is rarely seen except in cases associated with neurofibromatosis 1 (NF1) or in sites of prior radiation therapy. ${ }^{2,3,4} \mathrm{We}$ report a case of MPNST arising from a benign plexiform neurofibromatosis in a patient without NF1.

\section{Case Report:}

A 20 year lady presented to OPD with history of swelling at the low back since birth. Swelling was initially of about $5 \times 5 \mathrm{~cm}^{2}$ in size and had small tufts of hair. Swelling then, gradually increased in size since past $1-1.5$ years to a size of about $15 \times 15 \mathrm{~cm}^{2}$. There was no limb weakness. She had normal bowel and bladder habit. Family history was negative for von Recklinghausen's disease and on examination the patient did not show cutaneous stigmata of neurofibromatosis.

On examination, she had no neurological deficits. Local examination revealed diffuse swelling on the back in 


\section{Cose Report | Lamichhane B, et al. Large Malignant Peripheral Nerve Sheath Tumor}

lumbosacral region of approximately $15 \times 15 \mathrm{~cm}^{2}$ mass, soft with scarring of the overlying skin. Transillumination test was negative and cough reflex was absent. The swelling was adhered to the underlying tissue. There was no discharge from the swelling.

Magnetic Resonance Imaging (MRI) of lumbosacral spine showed mild degenerative changes in the lumbar spine with midline diffuse soft tissue lesion in posterior lumbar region which was highly suggestive of Plexiform neurofibroma. She was counseled for subtotal excision of the mass. At surgery, the findings were, highly vascular tumor encroaching the subcutaneous plane superficially and down to the interspinous process of vertebrae. Tumor was grayish white in color and amalgam of fibrotic and lipomatous component was present. Proper plane separating tumor and normal tissue was difficult to identify.

Post - operative period was uneventful except for the flap infection which was managed with regular Edingburg University Solution (EUSOL) dressing followed by secondary suturing later.

Histopathology of the mass showed markedly cellular circumscribed nodules in the diffuse neurofibromatous tumor tissue with expanded myxoneural profiles and spillage into adipose tissue and focally incorporating appendageal structures (Figure1).

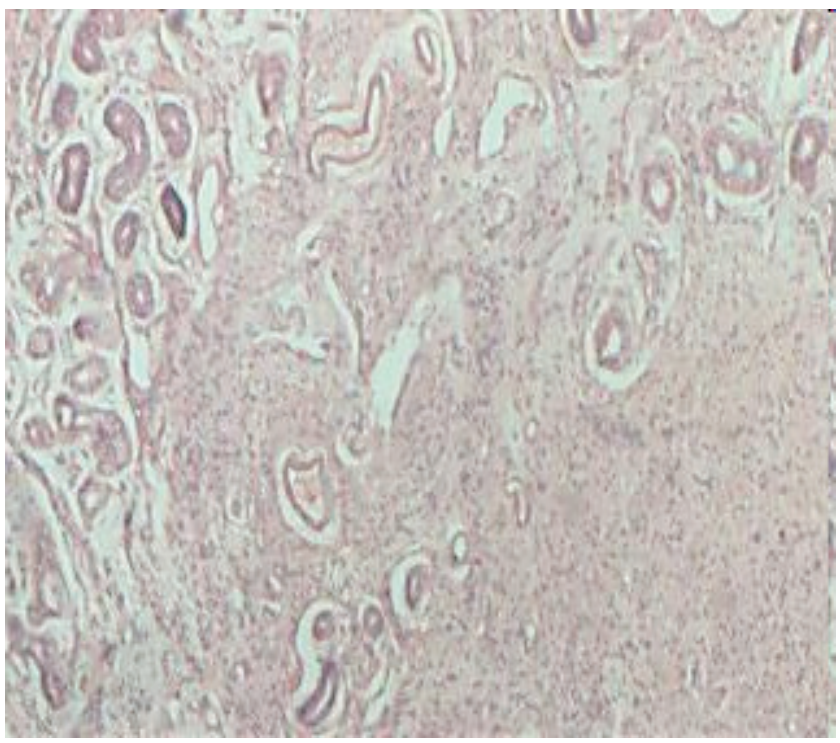

Figure 1: Appendigeal structure incorporated in diffuse areas

Cellular atypia in the form of nuclear enlargement and hyperchromasia with rare mitotic figures in the cellular areas with spindle tumor cells arranged in intersecting fascicular and stroiform patterns (Figure 2 and 3), giving it the impression of low grade malignant peripheral nerve sheath tumor (MPNST) arising in diffuse plexiform neurofibroma (Figure 4).

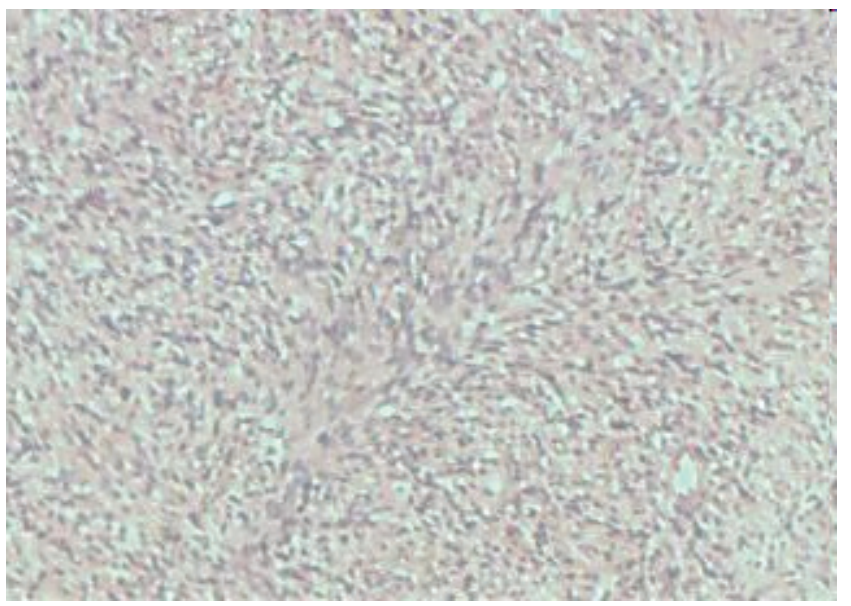

Figure 2: Cellular areas with storiform arrangement of tumor cells

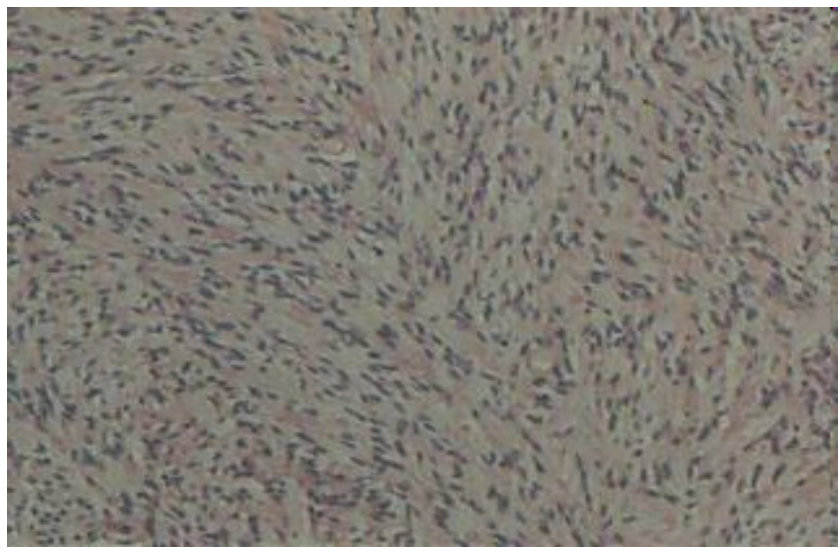

Figure 3: Fascicular arrangement of tumor cells

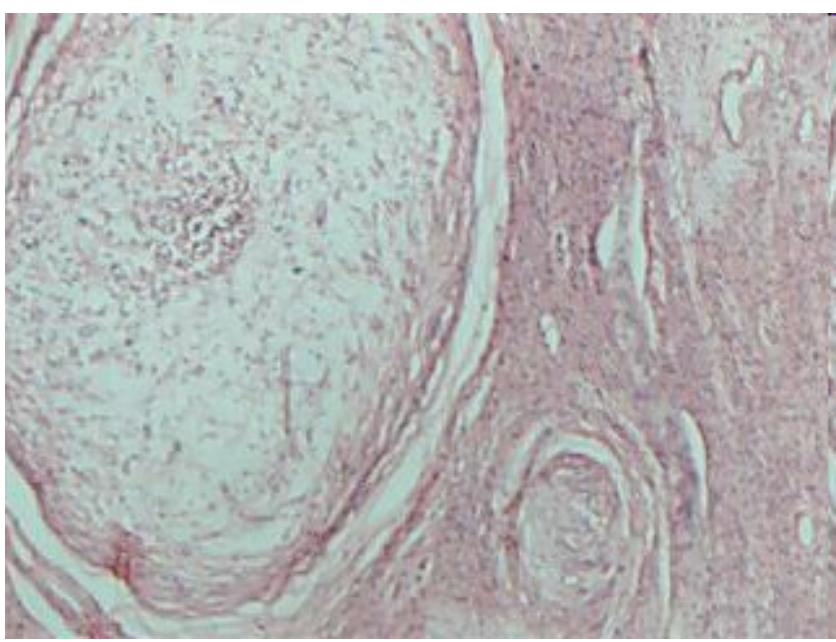

Figure 4: Myxoneural nodules of plexiform neurofibroma Patient was counseled and sent for postoperative radiotherapy and has been planned for regular follow- up. 


\section{Discussion:}

Malignant Peripheral Nerve Sheath Tumors of the lumbar spine are very rare tumors that may arise in a sporadic form or from a pre-existing neurofibroma associated with NF1. ${ }^{5}$ The most common sites are sciatic nerve, brachial plexus and sacral plexus. The diagnosis of these tumors remains problematic as it is based primarily on clinical suspicion. The majority of MPNST are composed of spindled cells arranged in a fascicular growth pattern similar to a fibrosarcoma, but fewer than 5\% of MPNSTs are either partially or purely epithelioid. At the low end of the grading spectrum (WHO grade II), MPNSTs merge with cellular neurofibromas, from which they are distinguished on the basis of increased cellularity, nuclear size $(>3 \mathrm{X}$ that of neurofibroma cells) and hyperchromasia. Also, epithelioid MPNST characterizes by increased cellularity, abundant cytoplasm and prominent nucleoli. The MPNSTs of NF1 are usually high grade or intermediate tumors. ${ }^{6-8}$ In our case, it was a low grade MPNST.

The following criteria are used for the histological diagnosis of MPNST - a) gross fusiform tumors in relation to nerves, b) microscopic features of spindle cell with fascicular pattern and varying degrees of mitosis, necrosis and tumor calcification, c) presence of associated benign neurofibroma or schwannanian cells, and d) positive immunohistochemical staining for S-100 protein, neuron specific enolase (NSE) and others like actin, cytokeratin (CK), smooth muscle actins (SMA), desmin, and vimentin to differentiate from other spindle cell sarcomas. ${ }^{9}$ A tumor may be termed MPNST if it has diffuse nuclear atypia and cellularity as in our case, it has diffuse nuclear atypia and extreme cellularity with backto-back cells or fascicles..$^{10}$ Immunohistochemical staining was however, not done in our case.

The 5-year survival rates in large series have been reported to range from 16 to $52 \%$. The reported 5-year survival rate for patients with MPNSTs without NF1 is as high as $50 \%$ and drops to as low as $10 \%$ in patients with MPNSTs and NF1. ${ }^{11}$

The treatment of MPNSTs represents a challenge. Surgical resection is the first line of therapy. The goal of operation is complete removal of the tumor with histologically clear margins of resection. This resection often requires en-bloc resection of major nerves and acceptance of potentially significant functional loss. Resectability rates are related to tumor location and range from 20\% (paraspinal) to $95 \%$ (extremity). ${ }^{12}$ MPNSTs have been demonstrated to have a higher local recurrence rate compared to other soft tissue sarcomas. Carli et al. have reported improved local control in MPNST patients treated with radiation therapy. This makes the use of radiation therapy conceptually attractive in patients with MPNST. ${ }^{13}$ Unfortunately, total resection of the tumor was not possible in our case, due to ill- defined margins and wide extension of the tumor. Thus, the patient was referred for radiation therapy and is being regularly followed up in our OPD.

Currently, postoperative radiotherapy is recommended by the Oncology Consensus Group13 as part of a uniform treatment policy for MPNSTs, much like other high-grade soft tissue sarcomas, even if clear surgical margins are obtained. Adjuvant radiotherapy should be administered wherever possible for intermediate to high-grade lesions and for lowgrade tumors after a marginal excision. ${ }^{14}$ Chemotherapy is not curative; therefore, its use is controversial.

\section{Conclusion:}

MPNST of the lumbar spine are very rare tumors that may arise in a sporadic form or from a pre-existing neurofibroma associated with NF1. Malignant transformation of preexisting Neurofibroma is even a rarer entity in absence of NF1 as in this case. Surgical resection with histologically clear margins with postoperative radiotherapy is the preferred line of therapy. Chemotherapy is usually ineffective and its role is controversial in such cases.

Acknowledgement: Photographs by courtesy of Dr. Panth R

\section{References:}

1. Hadiju S. Peripheral nerve sheath tumors. Histogenesis, classification, and prognosis. Cancer 1993;72:3549-52.

2. Mark RJ, Bailet JW, Poen J, et al. Post irradiation sarcoma of head and neck, Cancer 1993;72:887-93.

3. Ducatman BS, Scheithauer BW, Piepgras DG, et al. Malignant peripheral nerve sheath tumours: a clinicopathologic study of 120 cases. Cancer 1986;57:2006-21.

4. Rao UNM, Surti U, Hoffner L, et al. Cytogenetic and histologic correlation of peripheral nerve sheath tumors of soft tissue. Cancer Genet Cytogenet 1996;88:17-25.

5. King AA, Debaun MR, Riccardi VM, et al. Malignant Peripheral nerve sheath tumours in neurofibromatosis 1. Am J Med Genet 2000;93:388-92.

6. Koea JB, Leung D, Lewis JJ, et al. Histopathologic type: an independent prognostic factor in primary soft tissue sarcoma of the extremity. Ann Surg Oncol 
Case Report | Lamichhane B, et al. Large Malignant Peripheral Nerve Sheath Tumor

2003;10:432-40.

7. Louis DN, Ohgaki H, Wiestler OD, et al. WHO Classification of tumours of the central nervous system, $4^{\text {th }}$ edition, Lyon: IARC 2007, pp 160-2.

8. Stephen RG, John DR,Amir S, et al. Malignant peripheral nerve sheath tumor. J Surg Oncol 2008;97:340-9.

9. Trojani M, Contesso F, Coindre JM, et al. Soft tissue sarcomas in adults. Study of pathological prognostic variables and definition of histological grading system. Int J Cancer 1984;33:37-42.

10. Weiss SW, Goldblum JR. Enzinger and Weiss's Soft Tissue Tumors, St. Louis: Mosby Inc. 2001

11. Doorn PF, Molenaar WM, Bater J, et al. Malignant peripheral nerve sheath tumors in patients with and without neurofibromatosis. Eur J Surg Oncol 1995;21:78-82.

12. Baehring JM, Betensky RA, Batchelor TT. Malignant peripheral nerve sheath tumor : the clinical spectrum and outcome of treatment. Neurology 2003;61:696-8.

13. Carli M, Ferrari A, Mattke A, et al. Pediatric malignant peripheral nerve sheath tumor : The Italian and German soft tissue sarcoma cooperative group. J Clin Oncol 2005;23:8422-30.

14. Ferner RE, Gutmann DH: International consensus statement on malignant peripheral nerve sheath tumors in neurofibromatosis. Cancer Res 2002;62:1573-7. 